\title{
HERRAMIENTAS INFORMÁTICAS PARA EL ESTUDIANTE DE MEDICINA INVESTIGADOR
}

\section{COMPUTER TOOLS FOR MEDICAL STUDENT RESEARCHERS}

\section{Iván BARRIOS ${ }^{1,2}$, Ernesto BENÍTEZ ${ }^{2}$, René ROJAS ${ }^{2}$, Karen ZÁRATE ${ }^{2}$, Melissa LIRD 3 .}

${ }^{1}$ Estudiante de Matemática Estadística, Facultad de Ciencias Exactas y Naturales, Universidad Nacional de Asunción, San Lorenzo - Paraguay.

${ }^{2}$ Estudiante de Medicina y Cirugía, Facultad de Ciencias Médicas, Universidad Nacional de Asunción, San Lorenzo - Paraguay.

${ }^{3}$ Médica residente de Psiquiatría, Facultad de Ciencias Médicas, Universidad Nacional de Asunción, San Lorenzo - Paraguay.

Cómo citar este artículo: Barrios I, Benítez E, Rojas R, Zárate K, Lird M. Herramientas informáticas y oportunidades para el estudiante de medicina investigador. Medicina Clínica y Social. 2017;1(2):119125

\section{RESUMEN}

Las tecnologías de la información y la comunicación agrupan a todo el conjunto de técnicas y dispositivos empleados para el tratamiento y la transmisión de datos esto hace que el acceso a internet se haga cada vez más global; sin embargo, deben tenerse criterios bien claros sobre su uso para evitar acceder a contenido poco fiable. La búsqueda de información puede resumirse en tres pasos principales: análisis conceptual, elección de base de datos y preparación de la sintaxis de la búsqueda. Estos pasos deben ser respetados y conocidos en los principales motores de búsqueda científica, así también deben conocerse los estilos a la hora de redactar las referencias bibliográficas, lo que es mucho más sencillo con el uso de gestores de referencias. En esta revisión se podrá encontrar todos los conceptos considerados fundamentales para cualquiera que quiera iniciarse o profesionalizarse en la investigación, sobre todo en el pregrado de medicina.

Palabras clave: Investigación; Herramientas; Gestores bibliográficos; Estudiantes de Medicina.

\section{ABSTRACT}

Information and communication technologies bring together the whole set of techniques and devices used for the treatment and transmission of data, which makes Internet access increasingly more global; however, clear criteria must be taken on their use to avoid access to unreliable content. The search for information can be summarized in three main steps: conceptual analysis, database choice and preparation of the search syntax. These steps should be respected and known in the main scientific search engines, so the styles should be known when writing bibliographical references, which is much easier with the use of reference managers. In this review, it can find all the concepts considered fundamental for anyone who wants to start or become professionalized in research, especially in the medical undergraduate program.

Keywords: Research; Tools; Bibliographic managers; Medicine students. 


\section{INTRODUCCIÓN}

Las tecnologías de la información y la comunicación agrupan a todo el conjunto de técnicas y dispositivos empleados para el tratamiento y la transmisión de datos, este concepto engloba aquellos servicios basados en el intercambio de información, las redes de telecomunicaciones que dan soporte a dicho intercambio de datos y los terminales empleados para poder acceder a los distintos servicios (1).

Al acceso a internet se hace cada vez más global y ha tiendo efectos positivos en los campos de educación y salud ya que se ha demostrado que su uso mejora el acceso a la información, ayuda a la atención clínica y brinda soluciones en cuanto a investigación se trata (2). Vemos que muchas veces entre los médicos y estudiantes de medicina existen ciertas barreras que dificultan el acceso a estas tecnologías, sobre todo la falta de dominio del inglés y el desconocimiento de buscadores confiables (3).

Esta revisión pretende abarcar los puntos más resaltantes y actuales sobre aquellas herramientas informáticas que permiten al estudiante de medicina poder llevar a cabo con éxito las investigaciones que se proponga, por lo que nos centraremos en aquellas tecnologías que nos permiten buscar información, gestionar bibliografías, gestionar formularios o encuestas en línea.

Esto es de vital importancia teniendo en cuenta que los profesionales de la salud tienen muchos inconvenientes a la hora de realizar investigaciones, además de la poca cultura de investigar y publicar por parte de los estudiantes de medicina, por lo cual este trabajo puede ser de vital importancia para aquellos estudiantes que se están iniciando en la investigación (4-8).

\section{Herramientas de búsqueda bibliográfica}

Existe mucha información en internet sobre temas referentes a la salud, sin embargo, es fundamental realizar una lectura crítica de lo que encontramos y saber de dónde obtener información fidedigna, lo que constituye es uno de los principales obstáculos, ya que se utilizan buscadores generales como Google en lugar de los buscadores específicos para temas de salud $(9,10)$. Otro de los potenciales obstáculos lo constituye el idioma inglés, ya que existen varios alumnos que no manejan adecuadamente el idioma lo que dificulta el acceso a información certera y actualizada (11). Por lo anterior, un fundamental que en el pregrado se incluyan programas que ayuden a los estudiantes a encontrar información actualizada y confiable, no solo para su carrera como futuro investigador, sino como profesional (12).

La búsqueda de información puede resumirse en tres pasos principales: análisis conceptual, elección de base de datos y preparación de la sintaxis de la búsqueda (13). En el análisis conceptual se identifican los conceptos, se deciden aspectos que interesan de cada enfermedad a buscar; en la elección de la base de datos debemos tener en cuenta el ámbito geográfico, la cobertura temporal, el idioma y el tipo de documentos que podemos obtener; finalmente en la preparación de la sintaxis de búsqueda indagamos en diccionarios y tesauros la sintaxis correcta de los términos de búsqueda y la utilización de operadores booleanos $(13,14)$.

Los operadores booleanos son: AND, NOT y OR. El operador "AND" para obtención de documentos que contengan los dos términos, el operador "OR" para la obtención de documentos que contengan al menos uno de los términos y operador "NOT" para excluir documentos que 
contengan el término que aparece tras el "NOT" (15). Es importante utilizar correctamente estas herramientas para encontrar lo que realmente estamos buscando.

SCOPUS es la mayor base de datos de citas y resúmenes de literatura arbitrada y de fuentes de alta calidad en el Web (16). El idioma de esta base de datos es el inglés y existen estas modalidades de búsqueda: Basic search: ofrece posibilidad de realizar una consulta empleando criterios de selección, los resultados se pueden limitar por revista fuente, autor, tipo de documento y área. Autor search: permite recuperación de publicaciones de un autor, así como información sobre su afiliación e índice bibliométricos. Affiliation search: esta opción permite la búsqueda por el nombre de la organización y proporciona información sobre el país y la organización que pública $(16,17)$.

PUBMED es una base de datos bibliográfica de literatura médica, compilada por la National Library of Medicine (NLM) de los Estados Unidos, que incorpora detalles bibliográficos de artículos publicados en revistas de ciencias de la vida y libros on-line (18). Normalmente PubMed no proporciona el acceso al artículo completo, pero puede incorporar enlaces al sitio web de la editorial o al artículo en sí. Para realizar una búsqueda bibliográfica en PubMed hay que introducir un término o una frase con varios términos en la página de inicio de PubMed. Una vez realizada la búsqueda, se obtiene un listado de referencias, cada una de las cuales enlaza con los detalles bibliográficos del artículo.

Los términos MeSH (Medical Subject Headings) constituyen el vocabulario controlado (tesauro) para indexar las citas de PubMed. Hay términos MeSH mayores y menores (subheadings). Activando MeSH (pulsando en «MeSH Database») se puede verificar con facilidad cuál o cuáles son los términos MeSH más adecuados para realizar una búsqueda en esta base de datos (19).

GOOGLE SCHOLAR o Google Académico es un buscador creado por Google cuyo objetivo es la búsqueda de información especializada científica, médica y legal de carácter académico. La búsqueda en Google Académico es similar a la de Google, tras introducir los términos de búsqueda se muestra un listado de resultados en forma de direcciones web (18). Tiene funciones interesantes como herramientas que permiten medir el número de citas que tienen los investigadores y calcular sus índices de producción al igual que medir la productividad de las revistas, así como un muy efectivo buscador de artículos similares. Esta capacidad de seguimiento de citas y métricas ha sido mencionada en la literatura, centrando su potencial en la capacidad de producir métricas fiables (20).

LILACS es el acrónimo de Literatura Latinoamericana de Información en Ciencias de la Salud. Es una de las bases de datos más importantes de Bireme. Esta coordina, mantiene e indiza literatura relativa a Ciencias de la Salud, publicada en los países de la región desde el año 1980. BIREME es el acrónimo de Biblioteca Regional de Medicina, y conforma un sistema de bibliotecas que funciona como Centro Latino Americano y del Caribe de Información en Ciencias de la Salud.

De la misma forma que PubMed trabaja con términos llamados MeSH, en LILACS se trabaja con términos llamados DeCS, el cual es un vocabulario estructurado y trilingüe (portugués, castellano e inglés) creado por el Centro Latinoamericano y del Caribe de Información en Ciencias de la Salud, también conocido por su nombre original de Biblioteca Regional de Medicina 
(BIREME), es un centro especializado de la Organización Panamericana de la Salud / Organización Mundial de la Salud (OPS/OMS), disponibles en la Biblioteca Virtual en Salud (BVS). Se puede acceder a él de forma libre y gratuita (21).

Podemos concluir esta sección con los 10 pasos para realizar una correcta búsqueda bibliográfica, una propuesta del Grupo de Estudios Documentales de la Fundación Index en España, que podemos apreciar en la tabla 1 (22).

\section{TABLA 1. BÚSQUEDA BIBLIOGRÁFICA EN 10 PASOS. GRUPO DE ESTUDIOS DOCUMENTALES DE LA FUNDACIÓN INDEX (ESPAÑA)}

1. Formula una pregunta documental y limita la búsqueda.

2. Prepara la búsqueda identificando palabras (palabras clave, descriptores).

3. Traduce la pregunta al lenguaje de un tesauro (descriptores).

4. Elige las fuentes documentales o bases de datos bibliográficas.

5. Ejecuta la búsqueda utilizando el formulario de búsqueda avanzada (operadores booleanos)

6. Evalúa el resultado de la búsqueda y selecciona los documentos.

7. Recupera los documentos primarios seleccionados (texto completo).

8. Analiza y selecciona los documentos recuperados.

9. Completa la selección de documentos mediante búsqueda inversa (búsqueda de autores citados)

10. Realiza una síntesis de los resultados finales.

\section{Gestores de referencias bibliográficas}

Los gestores de referencias bibliográficas son aplicaciones que permiten crear, almacenar y dar formato a referencias bibliográficas recuperadas durante la búsqueda de información para su posterior tratamiento (23). Una de las principales ventajas a la hora de utilizar estas aplicaciones radica en que podemos crear de forma automática una bibliografía en la mayoría de los estilos, entre ellos Vancouver, Harvard, APA y otros utilizados en diferentes revistas, por lo tanto, podemos adecuar nuestra bibliografía en forma automática de acuerdo a la revista en la que estamos interesados en publicar (24).

La mayoría de los gestores funcionan de manera similar con tres funciones básicas. Entrada de datos: insertar manualmente o importación automática. Organización de los datos: crear carpetas, ver referencias y recuperación de la información. Salida de datos: crear referencias bibliográficas, insertar citas en un texto y compartir referencias (23). Estas aplicaciones también pueden integrarse con gran parte de los procesadores de texto utilizados en la actualidad.

Dentro de las herramientas de pago las más utilizadas son RefWorks y EndNote, y dentro de las gratuitas se encuentran Zotero y Mendeley $(25,26)$. Daremos especial énfasis a la explicación de las funciones del programa Zotero teniendo en cuenta que es una aplicación gratuita y a la vez intuitiva.

Zotero es una aplicación y complemento libre que se integra a la mayoría de los navegadores actuales. Una de las ventajas de Zotero radica en ser un software libre, de código abierto y gratuito. Al ser añadido al navegador va detectando los metadatos de la página sobre la que estamos o del documento que tenemos abierto en el navegador y es capaz de importar los metadatos (27). 
La instalación de Zotero y su posterior integración tanto con el navegador como con nuestro procesador de textos no conlleva mayores dificultades. La aplicación nos permite crear una cuenta y sincronizar nuestro catálogo bibliográfico en internet, con lo cual podemos acceder a nuestras referencias bibliográficas desde cualquier computadora con Zotero colocando el usuario y contraseña creados. Existen numerosos manuales disponibles en internet para aprender a manejar esta herramienta (28).

\section{Aplicativos de Google Drive}

Google Drive es un servicio de almacenamiento virtual disponible para cualquier usuario de Gmail de forma gratuita; permite subir todo tipo de archivos, al mismo tiempo pueden encontrarse las secciones: documentos, hojas de cálculo, presentaciones y formularios. Los formularios son un muy buen recurso para realizar investigaciones a bajo costo.

Estos formularios incluyen respuestas tipo: texto (corto o párrafo), selección múltiple, casilla desplegable, casilla de verificación, cuadrículas. También podemos personalizar cada pregunta de forma que su respuesta sean obligatorias y de un formato establecido (por ejemplo: solo texto, solo número, solo correo electrónico, fecha, etc.). Luego de completar el diseño de nuestro formulario el mismo puede ser enviado por correo electrónico o podemos generar un enlace para compartir con nuestra posible muestra $(29,30)$.

\section{CONCLUSIONES}

Las herramientas informáticas deben estar totalmente presentes durante todo el proceso de enseñanza - aprendizaje de cualquier disciplina, pero con especial importancia en las ciencias de la salud, ya que la constante actualización y globalización de la información nos obliga a estar entrenados en los métodos de investigación, búsqueda bibliográfica, así como estar entrenados a utilizar aquellas herramientas que facilitan este proceso.

En nuestro país existe una muy escasa cultura de publicación, y esta es casi nula durante el pregado, esto debe cambiar y motivarse la investigación en esta etapa donde los estudiantes pueden interiorizarse con los rígidos modelos de investigación internaciones e ir creciendo en esta labor; por este motivo es fundamental conocer las herramientas y recursos que fueron discutidos en esta revisión.

\section{CONFLICTOS DE INTERÉS Y FUENTE DE FINANCIACIÓN}

Los autores declaran no poseer conflictos de interés. Fuente de financiación: ninguna.

\section{REFERENCIAS BIBLIOGRÁFICAS}

1. Altés J. Papel de las tecnologías de la información y la comunicación en la medicina actual. Semin Fund Esp Reumatol. 2013;14:31-35. https://doi.org/10.1016/i.semreu.2013.01.005

2. Vásquez-Silva L, Ticse R, Guerra-Castañon F, Alfaro-Carballido L. Acceso, uso y preferencias de las tecnologías de información y comunicación por médicos de un Hospital General del Perú. Rev Peru Med Exp Salud Pública. 2015;32(2):289-293.URL. 
3. Veloz-Martínez MG, Almanza-Velasco E, Uribe-Ravell JA, Libiend-Díaz González L, Quintana-Romero V, Alanís-López P. Uso de tecnologías en información y comunicación por médicos residentes de ginecología y obstetricia. Investig En Educ Médica. 2012;1(4):183-189. URL.

4. Rios-González CM. Escasa publicación científica en estudiantes de medicina de Paraguay. Educ Médica. 2016;17(2):80-81. http://dx.doi.org/10.1016/i.edumed.2016.03.005

5. Torales J, Barrios I, Viveros-Filártiga D, Giménez-Legal E, Samudio M, Aquino S, et al. Conocimiento sobre métodos básicos de estadística, epidemiología e investigación de médicos residentes de la Universidad Nacional de Asunción, Paraguay. Educ Médica [Internet]. 2017 [citado el 13 de noviembre de 2016]; Disponible en: http://www.elsevier.es/es-revista-educacion-medica-71-avance-resumen-conocimiento-sobre-metodos-basicos-estadistica-S1575181316300912

6. Fleitas R, Yudik I, Báez Pérez E, Fernández Morín J, García Suárez L. Necesidades de aprendizaje de residentes para búsqueda y referenciación bibliográfica del trabajo de terminación de la especialidad. Rev Médica Electrónica. 2016;38(2):132-144. URL.

7. González BAG, Elías DGR, Ortiz RAR, Rodríguez NL, Hidalgo MDCV. Intervención educativa para elevar el conocimiento sobre Alfabetización informacional en Residentes de especialidades médicas. MULTIMED Rev Médica Granma. 2017;19(6):1020-1034. URL.

8. Demirdjian G, Rodríguez S, Vassallo JC, Irazola V, Rodríguez J. Capacitación hospitalaria de profesionales pediátricos en investigación y gestión. Arch Argent Pediatría. 2017;115(1):58-64. http://dx.doi.org/10.5546/aap.2017.58

9. Galán-Rodas E, Egoavil MS. Herramientas de productividad para el profesional médico y la investigación. Acta Médica Peruana. 2013;30(3):143-147. URL.

10. Gamboa-Peñaranda C, Zamora-llarionov A, Galán-Rodas E. Acceso a información médica actualizada en Internet: retos de la medicina actual. Acta Médica Costarric. 2014;56(4):186-187. URL.

11. Fernández-Altuna M de los Á, Martínez del Prado A, Gutiérrez Rayón $D$, Arriarán Rodríguez $\mathrm{E}$, Toriz Castillo HA, Betancourt Cravioto $\mathrm{M}$, et al. Encontrar sin perderse: ¿se ha frustrado al buscar la información médica que necesita? Investig En Educ Médica. 2016;5(18):75-87. URL.

12. Valverde Grandal O, Reyes R, Ángel S. Propuesta de programa para la formación de competencias informacionales en estudiantes de pregrado de Estomatología. Rev Cuba Estomatol. 2017;54(1):1-13. URL.

13. Aleixandre-Benavent R, Alcaide GG, de Dios JG, Alonso-Arroyo A. Fuentes de información bibliográfica (I). Fundamentos para la realización de búsquedas bibliográficas. Acta Pediátrica Esp. 2011;69(3):131-136. URL.

14. Caycho T. Manual de investigación para estudiantes de Psicología. Psicol Conoc Soc. 2017;7(1):226-229. URL.

15. Moraga C J, Manterola D C, Cartes-Velásquez R, Urrutia V S. ¿Dónde y cómo buscar evidencia científica en medicina? Rev Chil Cir. 2014;66(5):502-507. http://dx.doi.org/10.4067/S0718-40262014000500018

16. Cañedo Andalia R, Rodríguez Labrada R, Montejo Castells M. Scopus: la mayor base de datos de literatura científica arbitrada al alcance de los países subdesarrollados. $\mathrm{ACl}-$ MED. 2010;21(3):270-282. URL.

17. Navarro-Molina C, González-Alcaide G, Bolaños-Pizarro M, González de Dios J, Aleixandre-Benavent R. Fuentes de información bibliográfica (VI). Obtención de literatura 
científica con la base de datos Scopus y los buscadores especializados Scirus y Google Académico. Acta Pediátrica Esp. 2011;69(10):467. URL.

18. Muñoz Núñez CF, Sendra Portero F. Google y PubMed para médicos: ¿cómo buscar información sin perderse? Radiología. 2013;55:37-46. https://doi.org/10.1016/j.rx.2013.02.002

19. Cañedo Andalia R, Peña Rodríguez K, Rodríguez Labrada R, Sánchez C, Mercedes O, Concepción Reyes E. Procedimiento perfeccionado para la búsqueda bibliográfica en PubMed-Medline a través de Hinari. ACIMED. 2010;21(4):346-375. URL.

20. Halevi G, Moed H, Bar-Ilan J. Suitability of Google Scholar as a source of scientific information and as a source of data for scientific evaluation-Review of the Literature. J Informetr. 2017;11(3):823-834. https://doi.org/10.1016/i.joi.2017.06.005

21. Valero JS, Wanden-Berghe RG, Castiel LD. Los lenguajes de indización en e-Salud: su aplicación a los documentos sobre trastornos de la conducta alimentaria. Salud Colect. 2011;7(Supl1):61-69. URL.

22. Amezcua M. La Búsqueda Bibliográfica en diez pasos. Index Enferm. 2015;24(1-2):14. http://dx.doi.org/10.4321/S1132-12962015000100028

23. Alonso-Arroyo A, González de Dios J, Navarro-Molina C, Vidal-Infer A, Aleixandre-Benavent R. Fuentes de información bibliográfica (XII). Gestores de referencias bibliográficas: generalidades. Acta Pediátrica Esp. 2012;70(5):211-216. URL.

24. Boretto JG. Gestores de referencias bibliográficas. Rev Asoc Argent Ortop Traumatol. 2012;77(3):170. URL.

25. Pardo JC, Martín SM, Dani L, de Tomás JFÁ. Gestores bibliográficos. Form Médica Contin En Aten Primaria. 2014;21(6):355-359. https://doi.org/10.1016/S11342072(14)70786-8

26. Morenikeji OO. Using referencing software: Mendeley. Niger J Technol Res. 2017;12(1):48-51. URL.

27. Martínez RA, Lorenzo IM, Castañeda MD, Quintana MIC. Zotero, más allá de un gestor bibliográfico. Una experiencia con los docentes y nuevas metas. Rev DIM. 2013;9(25):1-13. URL.

28. Idri N. Zotero Software: A Means of Bibliographic Research and Data Organisation; Teaching Bibliographic Research. Arab World Engl J. 2015;124-133. URL.

29. Cánovas Peña JS, Jornadas de Intercambio de Experiencias de Innovación Educativa en Estadística, editores. Experiencias docentes en estadística. Cartagena: Universidad Politécnica de Cartagena; 2014.

30. Baiges EB, Surroca NV. Valoración del uso de las herramientas colaborativas Wikispaces y Google Drive, en la educación superior. Edutec Rev Electrónica Tecnol Educ. 2015;(49):1-12. http://dx.doi.org/10.21556/edutec.2014.49.39 